\title{
On the linear independence of the values of Gauss hypergeometric function
}

\author{
by \\ Ville Merilä (Oulu)
}

1. Introduction. The aim of this paper is to investigate the linear independence of values of functions defined by the series

$$
{ }_{2} F_{1}\left(1, B ; C ; \gamma_{\nu}\right)=\sum_{k=0}^{\infty} \frac{(B)_{k}}{(C)_{k}} \gamma_{\nu}^{k}, \quad B, C \in \mathbb{Q}^{*} \backslash \mathbb{Z}^{-},
$$

with $\gamma_{\nu} \in \mathbb{K}^{*}, 1 \leq \nu \leq D$, where $\mathbb{K}$ is an algebraic number field.

The hypergeometric series ${ }_{2} F_{1}(1, B ; C ; z)$ satisfies the first order differential equation

$$
z(1-z) \frac{d}{d z} y(z)+(C-B z-1) y(z)+1-C=0
$$

with three regular singularities, $\{0,1, \infty\}$. It defines an analytic function in the unit disc but which, as is well known, may be continued along any path in $\mathbb{C} \backslash[1, \infty)$. The arithmetic properties of this series have been widely studied in particular interesting cases such as the binomial ([2], [3], [6], [8], [18]) and logarithmic function ([13], [14, [28, [29], [35]), as well as a part of a wider class of $G$-functions ([5], [9], [10], [12], [32], [33]; see also [4]). Naturally, the references above represent only a fraction of the works in the literature. Nevertheless, there are no linear independence results with explicit approximations for ${ }_{2} F_{1}(1, B ; C ; z)$ in the generality of this paper. In 34. Vasilenko employed first type Padé approximations to obtain the linear independence over $\mathbb{Q}$ of values (1.1) at distinct points, all sufficiently close to the origin (with respect to normal absolute value). Ivankov proposed in [16] a combination of an effective construction of a linear approximating form and Siegel-Shidlovskiıı's method to prove linear independence of more general hypergeometric $E$ - and $G$-functions, comprising also (1.1), over an

2010 Mathematics Subject Classification: Primary 11J72; Secondary 11J61.

Key words and phrases: Gauss hypergeometric series, linear independence, algebraic number field, valuation, nonvanishing determinant. 
imaginary (archimedean) quadratic field. For irrationality questions, general theorems are given in [15. In addition, note also the article [30] by Sorokin, based on the works [23]-[27] of Nikishin. In that paper the question of linear independence of values of a hypergeometric function connected to a certain Markov function is studied with lower bounds that depend only on the moduli of the coefficients of related linear forms.

In the first part of this paper we compute explicit second type Padé approximations for the set of functions $\left\{{ }_{2} F_{1}\left(1, B ; C ; \gamma_{\nu} z\right)\right\}, \nu=1, \ldots, D$, in the spirit of Stihl [31], an approach originating from Maier [19] and thereafter generalized by Chudnovsky [7]. See also [22], where linear independence is considered for values of functions, and their derivatives, deriving from the Heine series, a $q$-analogue of the Gauss series.

In Section 4 we show that these approximations are linearly independent (Theorem 4.2), i.e. a certain determinant formed by the approximation polynomials does not vanish identically. This is the most important contribution of this work since the method of proof is new, and it applies also to the case of Heine series considered in [22]. The essential ingredient of the Padé construction, the application of the binomial (or $q$-binomial) theorem, is also the foundation of the determinant consideration. Hence the method may be applied, at least in some cases, to prove the independence of approximations for more general hypergeometric and $q$-hypergeometric series presented in [20].

The last part of this work consists of analytic and arithmetic estimates of the approximations, together with the proofs of the remaining theorems and corollaries.

2. Notations and results. Let $\mathbb{K}$ be an algebraic number field of degree $\kappa$ over $\mathbb{Q}$. In particular, we write $\mathbb{K}=\mathbb{I}$ in the case of an imaginary quadratic field. For a finite place $v$ of $\mathbb{K}$ over the prime $p$, we use the notation $v \mid p$, whereas we write $v \mid \infty$ for the infinite place. Let us normalize the absolute value $|*|_{v}$ of $\mathbb{K}$ so that if $v \mid p$, then $|p|_{v}=p^{-1}$, and if $v \mid \infty$, then $|x|_{v}=|x|$ for $x \in \mathbb{Q}$. Here, by $|*|$ we mean the usual absolute value in $\mathbb{Q}$. We shall adopt the notation

$$
\|*\|_{v}=|*|_{v}^{\kappa_{v} / \kappa} \quad \text { with } \quad \kappa_{v}=\left[\mathbb{K}_{v}: \mathbb{Q}_{v}\right],
$$

where $\mathbb{K}_{v}$ is the completion of $\mathbb{K}$ with respect to $v$. Now, for every $\alpha \in \mathbb{K}^{*}$ we have the product formula

$$
\prod_{w \in \mathcal{M}}\|\alpha\|_{w}=1
$$

where $\mathcal{M}_{\mathbb{K}}=\mathcal{M}$ is the set of places on $\mathbb{K}$. The height $H(\alpha)$ of $\alpha \in \mathbb{K}$ is defined by the equation 


$$
H(\alpha)=\prod_{w \in \mathcal{M}}\|\alpha\|_{w}^{*}, \quad\|\alpha\|_{w}^{*}=\max \left\{1,\|\alpha\|_{w}\right\},
$$

and more generally for a vector $\bar{B}=\left(b_{0}, \ldots, b_{D}\right) \in \mathbb{K}^{D+1}$ by

$$
H(\bar{B})=\prod_{w \in \mathcal{M}}\|\bar{B}\|_{w}^{*}, \quad\|\bar{B}\|_{w}^{*}=\max \left\{1,\|\bar{B}\|_{w}\right\},
$$

where

$$
|\bar{B}|_{w}=\max _{0 \leq i \leq D}\left|b_{i}\right|_{w}, \quad \text { thus } \quad\|\bar{B}\|_{w}=\max _{0 \leq i \leq D}\left\|b_{i}\right\|_{w} .
$$

Let $\xi_{i} \in \mathbb{K}_{v}, 0 \leq i \leq D$, be linearly independent over the field $\mathbb{K}$. Then, by a linear independence measure of the numbers $\xi_{0}, \ldots, \xi_{D}$, we mean an exponent $\mu>0$ in the lower bound

$$
\left|b_{0} \xi_{0}+\cdots+b_{D} \xi_{D}\right|_{v}>H^{-\mu-\varepsilon} \quad \forall \varepsilon>0,
$$

for every $\bar{B}=\left(b_{0}, \ldots, b_{D}\right) \in \mathbb{K}^{D+1} \backslash\{\overline{0}\}$ and $H=\max \left\{H(\bar{B}), H_{0}\right\}$, where $H_{0}=H_{0}(\varepsilon) \in \mathbb{Z}^{+}$is an effectively computable constant. When $D=1$, we set $\xi_{1}=1, b_{0}=1$ and call $\mu$ an irrationality measure of $\xi_{0}$, i.e.

$$
\left|\xi_{0}+b_{1}\right|_{v}>H^{-\mu-\varepsilon} \quad \forall \varepsilon>0,
$$

with $\bar{B}=\left(1, b_{1}\right) \in \mathbb{K}^{2}$ and $H=\max \left\{H\left(b_{1}\right), H_{0}\right\}, H_{0} \in \mathbb{Z}^{+}$.

As is customary, we make use of the Pochhammer notation

$$
(a)_{0}=1, \quad(a)_{n}=a(a+1) \cdots(a+n-1)
$$

for the generalized factorial, and more generally we denote

$$
(a ; b)_{0}=1, \quad(a ; b)_{n}=a(a+b) \cdots(a+b(n-1))
$$

for every $n \in \mathbb{Z}^{+}$.

Let $B, C \in \mathbb{Q}^{*}$, say $B=a / b, C=c / d, b / d=f / g, a, b, c, d, f, g \in \mathbb{Z}$, $b, d \geq 1, \operatorname{gcd}(a, b)=\operatorname{gcd}(c, d)=\operatorname{gcd}(f, g)=1$. In addition, for $v \mid p$, let $\operatorname{ord}_{p}(d) \geq \operatorname{ord}_{p}(b)$ and $\delta_{v}=0$, while $\delta_{v}=1$ for $v \mid \infty$. We shall also write

$$
E_{m}(a, b)=\operatorname{lcm}\{a, a+b, \ldots, a+b(m-1)\}, \quad \omega_{n}(d)=\prod_{p \mid d} p^{\operatorname{ord}_{p}(n !)}
$$

for $m, n \in \mathbb{Z}^{+}$and define

$$
\mu_{d}=\prod_{p \mid d} p^{1 /(p-1)}, \quad \lambda(b)=\frac{b}{\phi(b)} \sum_{\substack{i=1 \\ \operatorname{gcd}(i, b)=1}}^{b} \frac{1}{i}, \quad b, d \in \mathbb{Z}^{+},
$$

with $\phi$ the Euler totient function. We can now state the following theorem.

TheOREM 2.1. Let $\gamma_{\nu} \in \mathbb{K}^{*}, 1 \leq \nu \leq D$, be distinct algebraic numbers and $|\bar{\Gamma}|_{v}<1$ where $\bar{\Gamma}=\left(\gamma_{1}, \ldots, \gamma_{D}\right)$. If

$$
\log |\bar{\Gamma}|_{v}^{-1}>\frac{\left(\kappa-\kappa_{v} \delta_{v}\right) \log \mathcal{C}+\kappa(D+\eta) \log H(\bar{\Gamma})+\kappa_{v} \delta_{v} \log \mathcal{C}_{r}}{\kappa_{v}(D+\eta+1)}
$$


with $\eta \in \mathbb{R}^{+} \cup\{0\}$ and

$$
\mathcal{C}=5^{D} 4^{\eta+1} d \mu_{d}\left(g \mu_{g} e^{\lambda(b)}\right)^{D+\eta}, \quad \mathcal{C}_{r}=3^{D} 2^{\eta+1} d \mu_{d}\left(g \mu_{g} e^{\lambda(b)}\right)^{D+\eta},
$$

then the set of $D+1$ numbers $\left\{1,{ }_{2} F_{1}\left(1, B ; C ; \gamma_{1}\right), \ldots,{ }_{2} F_{1}\left(1, B ; C ; \gamma_{D}\right)\right\}$ is linearly independent over $\mathbb{K}$ with a linear independence measure

$$
\mu \leq \frac{\kappa(D+\eta+1) \log |\bar{\Gamma}|_{v}-\kappa \delta_{v} \log \left((5 / 3)^{D} 2^{\eta+1}\right)}{\left(\kappa-\kappa_{v} \delta_{v}\right) \log \mathcal{C}+\kappa(D+\eta) \log H(\bar{\Gamma})+\kappa_{v}(D+\eta+1) \log |\bar{\Gamma}|_{v}+\kappa_{v} \delta_{v} \log \mathcal{C}_{r}} .
$$

Corollary 2.2. Let $\gamma_{\nu} \in \mathbb{I}^{*}$ be such that 2.2 holds. Then, for $\bar{B} \in$ $\mathbb{Z}_{\mathbb{I}}^{D+1} \backslash\{\overline{0}\}$,

$$
\mu \leq-\frac{(D+\eta) \log H(\bar{\Gamma})+\log \mathcal{C}}{\log \mathcal{C}_{r}+(D+\eta) \log H(\bar{\Gamma})+(D+\eta+1) \log |\bar{\Gamma}|} .
$$

In particular, let $\gamma_{\nu}=\xi_{\nu} / q$ with $\xi_{\nu} \in \mathbb{I}^{*}$ and $q \in \mathbb{Z}_{\mathbb{I}} \backslash\{0\}$. Choose $\eta=0$ and let

$$
|q|>2 d \mu_{d}|\bar{\Xi}|\left(3 g \mu_{g} e^{\lambda(b)}|\bar{\Xi}|_{*} H(\bar{\Xi})\right)^{D},
$$

where $\bar{\Xi}=\left(\xi_{1}, \ldots, \xi_{D}\right)$ and $|\bar{\Xi}|_{*}=\min \{1,|\bar{\Xi}|\}$. Then the numbers in the set

$$
\left\{1,{ }_{2} F_{1}\left(1, B ; C ; \xi_{1} / q\right), \ldots,{ }_{2} F_{1}\left(1, B ; C ; \xi_{D} / q\right)\right\}
$$

are linearly independent over $\mathbb{Z}_{\mathbb{I}}$ and

$$
\mu \leq D+\frac{\log \left(\mathcal{C C}_{r}^{D}\left(H(\bar{\Xi})|\bar{\Xi}|_{*}\right)^{D(D+1)}\right)}{\log |q|-\log \left(\mathcal{C}_{r}|\bar{\Xi}|_{*}^{D} H(\bar{\Xi})^{D}|\bar{\Xi}|\right)}=D+\varepsilon(|q|) .
$$

Further, if $\xi_{\nu} \in \mathbb{Z}_{\mathbb{I}}$, then condition 2.3) becomes

$$
|q|>\mathcal{C}_{r}|\bar{\Xi}|^{D+1},
$$

and in the case of the logarithmic function $(B=1, C=2)$ over $\mathbb{K}=\mathbb{Q}$,

$$
s>3^{D} 2^{\eta+1} e^{D+\eta}|r|^{D+\eta+1}, \quad \eta \geq 0,
$$

with $\gamma_{\nu}=r_{\nu} / s, s>\max \left|r_{\nu}\right|=|r| \geq 1$. When (2.4) holds, then the numbers

$$
1, \log \left(1-r_{1} / s\right), \ldots, \log \left(1-r_{D} / s\right)
$$

are linearly independent over $\mathbb{Z}$ with

$$
\mu \leq \frac{\log \left(5^{D} 4^{\eta+1} e^{D+\eta}\right)+(D+\eta) \log s}{\log s-\log \left(3^{D} 2^{\eta+1} e^{D+\eta}\right)-(D+\eta+1) \log |r|} .
$$

Corollary 2.3. Consider $v \mid p$ and let $\gamma_{\nu}=r_{\nu} / s \in \mathbb{Q}^{*}, \operatorname{gcd}\left(r_{\nu}, s\right)=1$, $\max \left|r_{\nu}\right|=|r|,|\bar{\Gamma}|_{p}<1, \bar{B}=\left(b_{0}, \ldots, b_{D}\right) \in \mathbb{Z}^{D+1} \backslash\{\overline{0}\}$. If

$$
\log |\bar{\Gamma}|_{p}^{-1}>\frac{\log \left(5^{D} 4^{\eta+1} d \mu_{d}\left(g \mu_{g} e^{\lambda(b)}\right)^{D+\eta}\right)+(D+\eta) \log \max \{s,|r|\}}{D+\eta+1},
$$


then the set $\left\{1,{ }_{2} F_{1}\left(1, B ; C ; r_{1} / s\right), \ldots,{ }_{2} F_{1}\left(1, B ; C ; r_{D} / s\right)\right\}$ of $p$-adic numbers is linearly independent over $\mathbb{Z}$ with

$$
\mu \leq \frac{(D+\eta+1) \log |\bar{\Gamma}|_{p}}{\log \left(5^{D} 4^{\eta+1} d \mu_{d}\left(g \mu_{g} e^{\lambda(b)} \max \{s,|r|\}\right)^{D+\eta}\right)+(D+\eta+1) \log |\bar{\Gamma}|_{p}} .
$$

An interesting choice of parameters is $B=-1 / q, q>0, C=1, \gamma_{\nu}=p^{m} \cdot r_{\nu}$, $r_{\nu} \in \mathbb{Z} \backslash\{0\}, m \geq 1, p \nmid r_{\nu},|\bar{\Gamma}|_{p}=p^{-m}$. Now, if

$$
\log p>\frac{\log \left(5^{D} 4 e^{\lambda(q) D}\right)+D \log |r|}{m} \quad(\eta=0),
$$

then

$$
\left|b_{0}+b_{1}\left(1-p^{m} r_{1}\right)^{1 / q}+\cdots+b_{D}\left(1-p^{m} r_{D}\right)^{1 / q}\right|_{p} \geq H^{-\mu-\varepsilon}
$$

with

$$
\begin{aligned}
\mu & \leq \frac{(D+1) m \log p}{m \log p-\log \left(5^{D} 4 e^{\lambda(q) D}\right)-D \log |r|} \\
& =D+1+\frac{(D+1) \log \left(5^{D} 4 e^{\lambda(q) D}|r|^{D}\right)}{m \log p-\log \left(5^{D} 4 e^{\lambda(q) D}|r|^{D}\right)}=D+1+\varepsilon_{p}(m)
\end{aligned}
$$

for $H=\max \left\{\left|b_{0}\right|, \ldots,\left|b_{D}\right|\right\} \geq H_{0}$.

REMARK 2.4. In [16] Ivankov employed effective Padé approximations and Siegel's method to obtain good lower bounds for linear forms in the values of hypergeometric functions. For ${ }_{2} F_{1}(1, B ; C ; z)$ the general theorem for hypergeometric $G$-functions implies the linear independence over $\mathbb{Z}_{\mathbb{I}}$ of the values

$$
1,{ }_{2} F_{1}\left(1, B ; C ; \xi_{1} / q\right), \ldots,{ }_{2} F_{1}\left(1, B ; C ; \xi_{D} / q\right)
$$

when $\xi_{\nu} \in \mathbb{I}^{*},|q|>e^{C_{1} D(D+1) \Omega}, C_{1}>0, q \in \mathbb{Z}_{\mathbb{I}}$ and

$$
\Omega \geq \max \left\{|A+1|,|B+1|,\left|\xi_{n}\right|, 1 /\left|\xi_{n}\right|, 1 /\left|\xi_{n}-\xi_{m}\right|\right\}, \quad n \neq m, \quad \Omega \geq 3 .
$$

However, note that the dependence of the lower bound of $|q|$ on the number of points in the linear form is of magnitude $e^{\mathcal{O}\left(D^{2}\right)}$, whereas in our Corollary 2.2 it is $e^{\mathcal{O}(D)}$ by the inequality 2.3 .

Galochkin showed in [12], as a special case of a more general theorem for a class of $G$-functions, that the set of numbers

$$
\left\{1, \ln \left(1-\frac{1}{q}\right), \ln \left(1+\frac{1}{q}\right)\right\}
$$

is linearly independent over $\mathbb{Q}$ for any natural number $q>q_{0}$ where $q_{0} \geq$ $25 e^{56 / 5}>e^{14}$ (at least). Corollary 2.2, on the other hand, implies the linear independence of the numbers in 2.5 when $b>18 e^{2}\left(<e^{5}\right)$. Note, however, that in [14] Hata shows, in particular, that $q \geq 54$ suffices for the linear independence of the values in (2.5). 
3. Second type Padé approximations. In the following we shall compute second type Padé approximations for the functions

$$
g_{\nu}(z)=\sum_{k=0}^{\infty} \frac{(B)_{k}}{(C)_{k}}\left(\gamma_{\nu} z\right)^{k}, \quad \nu=1, \ldots, D .
$$

Lemma 3.1. Let $\sigma_{i, l}, i=0, \ldots, D l$, be defined by the equation

$$
\prod_{t=1}^{D}\left(\gamma_{t}-w\right)^{l}=\sum_{i=0}^{D l} \sigma_{i, l} w^{i}
$$

Then the coefficient $\sigma_{i, l}$ may be expressed as a sum

$$
\sigma_{i, l}=(-1)^{i} \sum_{i_{1}+\cdots+i_{D}=i}\left(\begin{array}{c}
l \\
i_{1}
\end{array}\right) \cdots\left(\begin{array}{c}
l \\
i_{D}
\end{array}\right) \gamma_{1}^{l-i_{1}} \cdots \gamma_{D}^{l-i_{D}}
$$

for $i=0,1, \ldots, D l$.

Proof. Use the binomial identity

$$
\left(\gamma_{\nu}-w\right)^{l}=\sum_{i=0}^{l}(-1)^{i}\left(\begin{array}{l}
l \\
i
\end{array}\right) \gamma_{\nu}^{l-i} w^{i}
$$

Theorem 3.2. For all $l \in \mathbb{Z}^{+}, \lambda \in \mathbb{N}$, and $1 \leq \nu \leq D$,

$$
A_{l, \lambda}(z) g_{\nu}(z)-B_{l, \lambda}^{(\nu)}(z)=R_{l, \lambda}^{(\nu)}(z)
$$

where

$$
\begin{aligned}
& A_{l, \lambda}(z)=\sum_{i=0}^{D l} \sigma_{i, l} \frac{(C)_{i+\rho-D l}}{(B)_{i+\lambda}} z^{D l-i} \\
& B_{l, \lambda}^{(\nu)}(z)=\sum_{n=0}^{D l+\lambda-1} g_{l, \lambda, n}^{(\nu)} z^{n} \\
& R_{l, \lambda}^{(\nu)}(z)=\sum_{n=0}^{\infty} g_{l, \lambda, n}^{(\nu)} z^{n}
\end{aligned}
$$

with $\operatorname{ord}_{z=0} R_{l, \lambda}^{(\nu)}(z) \geq(D+1) l+\lambda=\rho+1$ and

$$
g_{l, \lambda, n}^{(\nu)}=\sum_{i=0}^{\min \{n, D l\}} \sigma_{D l-i, l} \frac{(C)_{\rho-i}(B)_{n-i}}{(B)_{D l-i+\lambda}(C)_{n-i}} \gamma_{\nu}^{n-i} .
$$

Proof. We set the common approximation (denominator) polynomial

$$
A_{l, \lambda}(z)=\sum_{i=0}^{D l} \sigma_{i, l} \frac{(C)_{i+\rho-D l}}{(B)_{i+\lambda}} z^{D l-i}
$$


with $\rho=l+D l+\lambda-1$. By the Cauchy product, the coefficients of

$$
A_{l, \lambda}(z) g_{\nu}(z)=\sum_{n=0}^{\infty} g_{l, \lambda, n}^{(\nu)} z^{n}
$$

are determined as

$$
g_{l, \lambda, n}^{(\nu)}=\sum_{i=0}^{\min \{n, D l\}} \sigma_{D l-i, l} \frac{(C)_{\rho-i}(B)_{n-i}}{(B)_{D l-i+\lambda}(C)_{n-i}} \gamma_{\nu}^{n-i} .
$$

For the values $\lambda+D l \leq n \leq \rho$, we compute

$$
\begin{aligned}
g_{l, \lambda, n}^{(\nu)} & =\sum_{i=0}^{D l} \sigma_{i, l}(C+n-D l+i)_{\rho-n}(B+\lambda+i)_{n-D l-\lambda} \gamma_{\nu}^{n-D l+i} \\
& =\gamma_{\nu}^{n-D l} \sum_{j=0}^{l-1} p_{j} \sum_{i=0}^{D l} \sigma_{i, l} \gamma_{\nu}^{i} i^{j},
\end{aligned}
$$

where $p_{j}$ is defined by the equation

$$
(C+n-D l+i)_{\rho-n}(B+\lambda+i)_{n-D l-\lambda}=\sum_{j=0}^{l-1} p_{j} i^{j} .
$$

By applying the operator $\vartheta_{w}^{j}$, where $\vartheta_{w}=w(d / d w)$, to both sides of 3.2 , we obtain

$$
\sum_{i=0}^{D l} \sigma_{i, l} \gamma_{\nu}^{i} i^{j}=\vartheta_{w}^{j} \prod_{t=1}^{D}\left(\gamma_{t}-w\right)^{l}{ }_{\mid w=\gamma_{\nu}}=0 \quad \forall\left\{\begin{array}{l}
\nu=1, \ldots, D \\
j=0, \ldots, l-1 .
\end{array}\right.
$$

Therefore,

$$
g_{l, \lambda, n}^{(\nu)}=0 \quad \forall n=\lambda+D l, \ldots, \rho,
$$

and we set

$$
\begin{aligned}
B_{l, \lambda}^{(\nu)}(z) & :=\sum_{n=0}^{\lambda+D l-1} g_{l, \lambda, n}^{(\nu)} z^{n} \\
& =\sum_{n=0}^{\lambda+D l-1} \sum_{i=0}^{\min \{n, D l\}} \sigma_{D l-i, l} \frac{(C+n-i)_{\rho-n}}{(B+n-i)_{D l+\lambda-n}} \gamma_{\nu}^{n-i} z^{n}, \\
R_{l, \lambda}^{(\nu)}(z) & :=\sum_{n=\rho+1}^{\infty} g_{l, \lambda, n}^{(\nu)} z^{n}=z^{\rho+1} \sum_{k=0}^{\infty} g_{l, \lambda, \rho+k+1}^{(\nu)} z^{k}
\end{aligned}
$$

in order to get

$$
A_{l, \lambda}(z) g_{\nu}(z)-B_{l, \lambda}^{(\nu)}(z)=R_{l, \lambda}^{(\nu)}(z), \quad \nu=1, \ldots, D .
$$


4. The nonvanishing determinant. In this section we provide the proof for the independence of the approximations computed in Section 3. The core of the proof is Theorem 4.2 concerning the order of the determinant as a polynomial in $z$ connected to the systems of approximations. This promptly yields the validity of Theorem 4.3, i.e. the nonvanishing of the determinant. First, let us introduce a useful lemma the proof of which goes back to [21] (see also [1] and [17] for further discussions on generalized Vandermonde determinants).

Lemma 4.1. Let $n$ be a nonnegative integer, and let $B_{m}(x)$ denote an $n \times m$ matrix formed by applying $x(d / d x)$ subsequently on its columns:

$$
B_{m}(x)=\left[\begin{array}{ccccc}
1 & 0 & 0 & \ldots & 0 \\
x & x & x & \ldots & x \\
x^{2} & 2 x^{2} & 4 x^{2} & \ldots & 2^{m-1} x^{2} \\
\vdots & \vdots & \vdots & \ddots & \vdots \\
x^{i} & i x^{i} & i^{2} x^{i} & \ldots & i^{m-1} x^{i} \\
\vdots & \vdots & \vdots & \ddots & \vdots \\
x^{n-1} & (n-1) x^{n-1} & (n-1)^{2} x^{n-1} & \ldots & (n-1)^{m-1} x^{n-1}
\end{array}\right] .
$$

If $n=m_{1}+\cdots+m_{d}$ is a partition of $n$, then

$$
\operatorname{det}\left[B_{m_{1}}\left(\alpha_{1}\right) \ldots B_{m_{d}}\left(\alpha_{d}\right)\right]=\prod_{j=1}^{d} \alpha_{j}^{\left(\begin{array}{c}
m_{j} \\
2
\end{array}\right)} \prod_{i=1}^{m_{j}-1} i ! \prod_{1 \leq j<i \leq d}\left(\alpha_{i}-\alpha_{j}\right)^{m_{i} m_{j}} .
$$

TheOREM 4.2. If $\gamma_{n} \neq \gamma_{m} \neq 0$ for every $n \neq m, 1 \leq n, m \leq D$ and $C-B \neq 0,-1,-2, \ldots$, then

$$
\operatorname{det}\left[g_{l, \lambda+j-1, \rho+j}^{(\nu)}\right]_{j \nu} \neq 0, \quad 1 \leq j \leq D, 1 \leq \nu \leq D,
$$

where $g_{l, \lambda+j-1, \rho+j}^{(\nu)}$ is the coefficient of the lowest term in $R_{l, \lambda+j-1}^{(\nu)}(z)$.

Proof. Firstly, let us compute

$$
\begin{aligned}
g_{l, \lambda, \rho+1}^{(\nu)} & =\gamma_{\nu}^{l+\lambda} \sum_{i=0}^{D l} \sigma_{i, l} \frac{(B+\lambda+i)_{l}}{C+\lambda+i+l-1} \gamma_{\nu}^{i} \\
& =\gamma_{\nu}^{l+\lambda} \sum_{i=0}^{D l} \sigma_{i, l} \frac{(B+\lambda+i)_{l-1}(C+\lambda+i+l-1+B-C)}{C+\lambda+i+l-1} \gamma_{\nu}^{i} \\
& =\gamma_{\nu}^{l+\lambda} \sum_{i=0}^{D l} \sigma_{i, l}(B+\lambda+i)_{l-1}\left(1+\frac{B-C}{C+\lambda+i+l-1}\right) \gamma_{\nu}^{i}
\end{aligned}
$$




$$
\begin{aligned}
& =\gamma_{\nu}^{l+\lambda} \sum_{i=0}^{D l} \sigma_{i, l}(B+\lambda+i)_{l-1} \gamma_{\nu}^{i}+(B-C) \sum_{i=0}^{D l} \sigma_{i, l} \frac{(B+\lambda+i)_{l-1}}{C+\lambda+i+l-1} \gamma_{\nu}^{i+l+\lambda} \\
& =(B-C) \gamma_{\nu}^{l+\lambda} \sum_{i=0}^{D l} \sigma_{i, l} \frac{(B+\lambda+i)_{l-1}}{C+\lambda+i+l-1} \gamma_{\nu}^{i}=\cdots \\
& =(-1)^{l}(C-B)_{l} \gamma_{\nu}^{l+\lambda} \sum_{i=0}^{D l} \frac{\sigma_{i, l}}{C+\lambda+i+l-1} \gamma_{\nu}^{i},
\end{aligned}
$$

by applying (3.7) repeatedly. Let us introduce an auxiliary variable $x$, denote

$$
G^{(\nu)}(\lambda, x)=\left(x \gamma_{\nu}\right)^{\lambda} \sum_{i=0}^{D l} \sigma_{i, l} \frac{\left(\gamma_{\nu} x\right)^{i}}{C+\lambda+i+l-1}
$$

and form the matrix

$$
\Omega(x)=\left[\begin{array}{ccc}
G^{(1)}(\lambda, x) & \ldots & G^{(D)}(\lambda, x) \\
\vdots & \ddots & \vdots \\
G^{(1)}(\lambda+j, x) & \ldots & G^{(D)}(\lambda+j, x) \\
\vdots & \ddots & \vdots \\
G^{(1)}(\lambda+D-1, x) & \ldots & G^{(D)}(\lambda+D-1, x)
\end{array}\right] .
$$

Thus,

$$
\operatorname{det}\left[g_{l, \lambda+j-1, \rho+j}^{(\nu)}\right]_{j \nu}=(-1)^{D l}(C-B)_{l}^{D}\left(\gamma_{1} \cdots \gamma_{D}\right)^{l} \operatorname{det} \Omega(1) .
$$

As a polynomial in $x$, we have

$$
\begin{aligned}
\operatorname{det} \Omega(x) & =\left(\gamma_{1} \cdots \gamma_{D}\right)^{\lambda} x^{\left(\begin{array}{c}
D \\
2
\end{array}\right)+D \lambda}\left|\begin{array}{ccc}
F^{(1)}(0, x) & \ldots & F^{(D)}(0, x) \\
\vdots & \ddots & \vdots \\
F^{(1)}(D-1, x) & \ldots & F^{(D)}(D-1, x)
\end{array}\right| \\
& =\left(\gamma_{1} \cdots \gamma_{D}\right)^{\lambda} x^{\left(\begin{array}{c}
D \\
2
\end{array}\right)+D \lambda} \operatorname{det} \widetilde{\Omega}(x)
\end{aligned}
$$

with

$$
F^{(\nu)}(j, x)=\gamma_{\nu}^{j} \sum_{i=0}^{D l} \sigma_{i, l} \frac{\left(\gamma_{\nu} x\right)^{i}}{C+\lambda+i+j+l-1}, \quad 0 \leq j \leq D-1 .
$$

If $\gamma_{n} \neq \gamma_{m}, n \neq m$, then $\operatorname{det} \widetilde{\Omega}(0)$ is a nonzero Vandermonde determinant times $\left(\gamma_{1} \cdots \gamma_{D}\right)^{D l} /(C+\lambda+l-1)_{D}$, and hence

$$
\operatorname{ord}_{x=0} \operatorname{det} \Omega(x)=\left(\begin{array}{l}
D \\
2
\end{array}\right)+D \lambda .
$$


In particular, $\operatorname{det} \Omega(x) \neq 0$ (as a polynomial of $x$ ). Consider next the following $D \times D(l+1)$ and $D l \times D(l+1)$ matrices:

$$
A:=\left[\begin{array}{cccccccc}
\frac{\sigma_{0, l}}{C+\lambda+l-1} & \frac{\sigma_{1, l}}{C+\lambda+l} & \cdots & \frac{\sigma_{D l, l}}{C+\lambda+D l+l-1} & 0 & 0 & \ldots & 0 \\
0 & \frac{\sigma_{0, l}}{C+\lambda+l} & \cdots & \frac{\sigma_{D l-1, l}}{C+\lambda+D l+l-1} & \frac{\sigma_{D l, l}}{C+\lambda+D l+l} & 0 & \ldots & 0 \\
\vdots & \vdots & \ddots & \vdots & \vdots & \vdots & \ddots & \vdots \\
0 & \ldots & 0 & \frac{\sigma_{0, l}}{C+\lambda+D+l-2} & \ldots & \ldots & & \frac{\sigma_{D l, l}}{C+\lambda+D l+D+l-2}
\end{array}\right]
$$

and $B=\left[B_{1} \ldots B_{k} \ldots B_{l}\right]^{T}$ (a column vector with matrix entries), where

$$
B_{k}=\left[b_{i j}\right]_{D \times D(l+1)}, \quad b_{i j}= \begin{cases}0, & 1 \leq j \leq i-1, \\ \sigma_{j-i, k}, & i \leq j \leq i+D k, \\ 0, & i+D k<j \leq D(l+1) .\end{cases}
$$

Set $\Delta=\left[\begin{array}{ll}A & B\end{array}\right]^{T}$. Then

$$
\Delta V(x)=\left[\begin{array}{l}
A \\
B
\end{array}\right] V(x)=\left[\begin{array}{ll}
\widehat{\Omega}(x) & O \\
X(x) & E
\end{array}\right],
$$

where by (3.7), $O$ is a $D \times D l$ zero matrix and $X(x)$ a $D l \times D$ matrix such that $X(1)$ is zero, while $\operatorname{det} \widehat{\Omega}(x)=x^{\left(\begin{array}{c}D \\ 2\end{array}\right)} \operatorname{det} \widetilde{\Omega}(x)$. Furthermore,

$$
V(x)=\left[\begin{array}{llll}
V_{0}(x) & V_{1} & \ldots & V_{D}
\end{array}\right],
$$

where

$$
V_{0}(x)=\left[\begin{array}{ccc}
1 & \cdots & 1 \\
\gamma_{1} x & \cdots & \gamma_{D} x \\
\vdots & \ddots & \vdots \\
\left(\gamma_{1} x\right)^{D l+D-1} & \cdots & \left(\gamma_{D} x\right)^{D l+D-1}
\end{array}\right]
$$

and

$$
V_{\nu}=\left[(C+\lambda+i+l-1) i^{j} \gamma_{\nu}^{i}\right]_{i j}, \quad 0 \leq i \leq D l+D-1,0 \leq j \leq l-1,
$$

is a $(D l+D) \times l$ matrix. The entries of $V$ are polynomials in $i$ and after elementary column operations (repeated cancellations of lower terms of the polynomials, i.e. the terms $(C+\lambda+l-1) i^{j} \gamma_{\nu}^{i}$ in $\left.V_{\nu}\right)$, Lemma 4.1 yields

$$
\operatorname{det} V(1)=\prod_{t=1}^{D} \gamma_{t}^{\left(\begin{array}{c}
l+1 \\
2
\end{array}\right)} \prod_{j=1}^{l} j ! \prod_{1 \leq n<m \leq D}\left(\gamma_{m}-\gamma_{n}\right)^{(l+1)^{2}} \neq 0
$$

when $\gamma_{t} \neq 0$ and $\gamma_{m} \neq \gamma_{n}$. Since $\operatorname{rank} B=D l$, it follows that $\operatorname{rank} E=D l$ ( $E$ is independent of $x$ ), and thus by (4.2),

$$
\operatorname{det} \Delta \operatorname{det} V(x)=\operatorname{det} E \operatorname{det} \widehat{\Omega}(x), \quad \operatorname{det} E \neq 0 .
$$

This implies that $\operatorname{det} \Delta=0$ if and only if $\operatorname{det} \widehat{\Omega}(1)=0$. However, $\operatorname{det} \widehat{\Omega}(x)$ $\neq 0$ (as a polynomial in $x)$, which yields $\operatorname{det} \Delta \neq 0($ does not depend on $x)$. 
Furthermore,

$$
\operatorname{det} \Omega(x)=\operatorname{det} \Delta \operatorname{det} E^{-1} x^{D \lambda} \operatorname{det} V(x) \prod_{t=1}^{D} \gamma_{t}^{\lambda},
$$

and in particular $\operatorname{det} \Omega(1) \neq 0$, which together with 4.1 proves the theorem.

TheOREM 4.3. If $\gamma_{n} \neq \gamma_{m} \neq 0$ for every $n \neq m, 1 \leq n, m \leq D$ and $C-B \neq 0,-1,-2, \ldots$, then

$$
\Delta_{l, \lambda}(z)=\left|\begin{array}{cccc}
-A_{l, \lambda}(z) & -A_{l, \lambda+1}(z) & \ldots & -A_{l, \lambda+D}(z) \\
B_{l, \lambda}^{(1)}(z) & B_{l, \lambda+1}^{(1)}(z) & \ldots & B_{l, \lambda+D}^{(1)}(z) \\
\vdots & \vdots & \ddots & \vdots \\
B_{l, \lambda}^{(\nu)}(z) & B_{l, \lambda+1}^{(\nu)}(z) & \ldots & B_{l, \lambda+D}^{(\nu)}(z) \\
\vdots & \vdots & \ddots & \vdots \\
B_{l, \lambda}^{(D)}(z) & B_{l, \lambda+1}^{(D)}(z) & \ldots & B_{l, \lambda+D}^{(D)}(z)
\end{array}\right|_{(D+1) \times(D+1)} \neq 0 .
$$

In particular,

$$
\operatorname{ord}_{z=0} \Delta_{l, \lambda}=\operatorname{deg}_{z} \Delta_{l, \lambda} .
$$

Proof. By (3.8), we may transform the determinant (4.3) into

$$
(-1)^{D+1} \Delta_{l, \lambda}(z)=\left|\begin{array}{cccc}
A_{l, \lambda}(z) & A_{l, \lambda+1}(z) & \ldots & A_{l, \lambda+D}(z) \\
R_{l, \lambda}^{(1)}(z) & R_{l, \lambda+1}^{(1)}(z) & \ldots & R_{l, \lambda+D}^{(1)}(z) \\
\vdots & \vdots & \ddots & \vdots \\
R_{l, \lambda}^{(\nu)}(z) & R_{l, \lambda+1}^{(\nu)}(z) & \ldots & R_{l, \lambda+D}^{(\nu)}(z) \\
\vdots & \vdots & \ddots & \vdots \\
R_{l, \lambda}^{(D)}(z) & R_{l, \lambda+1}^{(D)}(z) & \ldots & R_{l, \lambda+D}^{(D)}(z)
\end{array}\right| .
$$

Because

$$
\operatorname{ord}_{z=0} A_{l, \lambda+j}(z)=0 \quad \text { and } \quad \operatorname{ord}_{z=0} R_{l, \lambda+j}^{(\nu)}(z) \geq \rho+j+1, \quad j=0, \ldots, D,
$$

where $\rho=(D+1) l+\lambda-1$, we notice that

$$
\operatorname{ord}_{z=0} \Delta_{l, \lambda}(z)=\rho \cdot D+\left(\begin{array}{c}
D+1 \\
2
\end{array}\right)
$$

exactly when

$$
\begin{aligned}
& \operatorname{det}\left[g_{l, \lambda+j-1, \rho+j}^{(\nu)}\right]_{j \nu}=(-1)^{D l}(C-B)_{l}^{D} \prod_{t=1}^{D} \gamma_{t}^{l} \operatorname{det} \Omega(1) \neq 0, \\
& 1 \leq \nu, j \leq D .
\end{aligned}
$$


On the other hand, as

$$
\operatorname{deg}_{z} A_{l, \lambda+j}(z)=D l \quad \text { and } \quad \operatorname{deg}_{z} B_{l, \lambda+j}^{(\nu)} \leq \lambda+D L+j-1,
$$

it follows from 4.3 that

$$
\operatorname{deg}_{z} \Delta_{l, \lambda}(z) \leq \rho \cdot D+\left(\begin{array}{c}
D+1 \\
2
\end{array}\right) .
$$

Thus, if (4.5) is different from zero, then $\operatorname{ord}_{z} \Delta_{l, \lambda}=\operatorname{deg}_{z} \Delta_{l, \lambda}$.

\section{Estimates for the approximation polynomials and for the remainder}

Lemma 5.1. Let $c, d \in \mathbb{Z} \backslash\{0\}$ with $\operatorname{gcd}(c, d)=1$ and $p \in \mathbb{P}$ be such that $p \nmid d$. If $p^{m} \mid i$ !, then $p^{m} \mid(c+d k ; d)_{i}$ for $i, m \in \mathbb{Z}^{+}$.

Proof. Since $p \nmid d$, there exists $d^{-1} \in \mathbb{Z}$ such that $d d^{-1} \equiv 1\left(\bmod p^{m}\right)$. Thus,

$$
\begin{aligned}
& (c+d k)(c+d(k+1)) \cdots(c+d(k+i-1)) \\
& \equiv d^{i}\left(c d^{-1}+k\right)\left(c d^{-1}+k+1\right) \cdots\left(c d^{-1}+k+i-1\right) \\
& =d^{i}\left(\begin{array}{c}
c d^{-1}+k+i-1 \\
i
\end{array}\right) i ! \equiv 0\left(\bmod p^{m}\right)
\end{aligned}
$$

by the hypothesis $p^{m} \mid i$, and so $p^{m} \mid(c+d k ; d)_{i}$.

As a consequence of Lemma 5.1 we have

$$
\prod_{p \mid d} p^{\operatorname{ord}_{p}(i !)} \frac{(c+d k ; d)_{i}}{i !} \in \mathbb{Z}, \quad \text { as } \quad i !=\prod_{p \nmid d} p^{\operatorname{ord}_{p}(i !)} \prod_{p \mid d} p^{\operatorname{ord}_{p}(i !)} .
$$

Note also that if $p \mid d$, then $p \nmid(c ; d)_{i}$, since $\operatorname{gcd}(c, d)=1$.

Let us denote the coefficients of the polynomial $A_{l, \lambda}(z)$ by $A_{i}=\sigma_{D l-i, l} a_{i}$, and the coefficients of $g_{\nu}(z)$ by $f_{k}^{(\nu)}=f_{k} \gamma_{\nu}^{k}$, i.e.

$$
a_{i}=\frac{(C)_{\rho-i}}{(B)_{D l+\lambda-i}}, \quad f_{k}=\frac{(B)_{k}}{(C)_{k}} .
$$

Then, for $0 \leq i \leq D l, 0 \leq i+k \leq D l+\lambda-1$,

$$
a_{i} f_{k}=\frac{(C)_{D l+\lambda+l-1-i}(B)_{k}}{(B)_{D l+\lambda-i}(C)_{k}}=\frac{(C+k)_{D l+\lambda+l-i-1-k}}{(B+k)_{D l+\lambda-i-k}}=\frac{(C+k)_{\rho-i-k}}{(B+k)_{D l+\lambda-i-k}} \text {. }
$$

The partial fraction decomposition

$$
\begin{aligned}
\frac{1}{(B+k)_{D l+\lambda-i-k}} & =\sum_{j=0}^{\rho-l-i-k} \frac{b \alpha_{j}}{a+b(k+j)}, \\
\alpha_{j} & =\frac{(-1)^{j}}{(\rho-l-i-k) !}\left(\begin{array}{c}
\rho-l-i-k \\
j
\end{array}\right),
\end{aligned}
$$


gives

$$
\begin{aligned}
& \frac{(C+k)_{\rho-i-k}}{(B+k)_{D l+\lambda-i-k}} \\
& \quad=\frac{(C+k)_{\rho-i-k}}{(\rho-l-i-k) !} \sum_{j=0}^{\rho-l-i-k}\left(\begin{array}{c}
\rho-l-i-k \\
j
\end{array}\right) \frac{(-1)^{j}}{B+k+j},
\end{aligned}
$$

where we write

$$
\frac{(C+k)_{\rho-i-k}}{(\rho-l-i-k) !}=l !\left(\begin{array}{c}
C+k+l-1 \\
l
\end{array}\right)\left(\begin{array}{c}
C+\rho-i-1 \\
\rho-l-i-k
\end{array}\right) .
$$

Altogether, we obtain the expression

$$
\frac{(C+k)_{\rho-i-k}}{(B+k)_{D l+\lambda-i-k}}=l !\left(\begin{array}{c}
C+k+l-1 \\
l
\end{array}\right)\left(\begin{array}{c}
C+\rho-i-1 \\
\rho-l-i-k
\end{array}\right) \frac{(\rho-l-i-k) !}{(B+k)_{D l+\lambda-i-k}},
$$

where, by (5.1) and the first definition in (2.1),

$$
E_{D l+\lambda-i}(a, b) \frac{(\rho-l-i-k) !}{(B+k)_{D l+\lambda-i-k}} \in \mathbb{Z} .
$$

More precisely, since $\operatorname{gcd}\left(b, E_{D l+\lambda-i}(a, b)\right)=\operatorname{gcd}(a, b)=1$, we have

$$
\frac{\prod_{p \nmid b} p^{\operatorname{ord}_{p}((\rho-l-i-k) !)}}{(a+b k ; b)_{D l+\lambda-i-k}} E_{D l+\lambda-i}(a, b) \in \mathbb{Z} .
$$

This, together with $\operatorname{gcd}(c, d)=1$, yields

$$
E_{D l+\lambda-i}(a, b) d^{l-2} g^{D l+\lambda-i-k} \omega_{l}(d) \omega_{\rho-l-i-k}(g) \frac{a_{i} f_{k}}{l !} \in \mathbb{Z},
$$

where $g$ is the denominator of $b / d$. Finally,

$$
E_{D l+\lambda}(a, b) d^{l-2} g^{D l+\lambda} \omega_{l}(d) \omega_{\rho-l}(g) \frac{a_{i} f_{k}}{l !} \in \mathbb{Z} \quad \forall 0 \leq i+k \leq D l+\lambda-1 .
$$

LEMMA 5.2. For the coefficients of the approximation polynomials of Theorem 3.2 we have

$$
\frac{\Omega_{l, \lambda}^{(D)}(a, b, d)}{l !} a_{i} f_{k} \in \mathbb{Z} \quad \forall 0 \leq i+k \leq D l+\lambda-1,0 \leq i \leq D l,
$$

and furthermore

$$
\frac{\Omega_{l, \lambda}^{(D)}(a, b, d)}{l !} A_{l, \lambda}(z), \frac{\Omega_{l, \lambda}^{(D)}(a, b, d)}{l !} B_{l, \lambda}^{(\nu)}(z) \in \mathbb{Z}\left[\gamma_{1}, \ldots, \gamma_{D}, z\right],
$$

where

$$
\Omega_{l, \lambda}^{(D)}(a, b, d)=d^{l-2} g^{D l+\lambda} \omega_{\rho-l}(g) \omega_{l}(d) E_{D l+\lambda}(a, b) .
$$

Proof. The coefficients of the polynomial $B_{l, \lambda}^{(\nu)}(z)$ are

$$
g_{l, \lambda, n}^{(\nu)}=\sum_{i+k=n} A_{i} f_{k}^{(\nu)}=\sum_{i+k=n} \sigma_{D l-i, l} \gamma_{\nu}^{k} a_{i} f_{k}
$$


for $0 \leq n \leq D l+\lambda-1$, and

$$
\frac{d^{l-2} g^{D l+\lambda} \omega_{l}(d) \omega_{\rho-l}(g) E_{D l+\lambda}(a, b)}{l !} \cdot a_{i} f_{k} \in \mathbb{Z},
$$

as can be seen by the previous discussion.

Lemma 5.3. If $\lambda=\lambda(l)=\lfloor\eta l\rfloor, \eta \geq 0$, then for every $w \in \mathcal{M}$ and $l$ sufficiently large,

$$
\begin{aligned}
& \max \left\{\left|\left(\Omega_{l, \lambda}^{(D)}(a, b, d) / l !\right) A_{l, \lambda}(z)\right|_{w},\left|\left(\Omega_{l, \lambda}^{(D)}(a, b, d) / l !\right) B_{l, \lambda}^{(\nu)}(z)\right|_{w}\right\} \\
& \leq \mathcal{C}^{\delta_{w} l}\left(|\overline{\Gamma(z)}|_{w}^{D+\eta}\right)^{l+c_{1} \log l},
\end{aligned}
$$

where $c_{1}>0$ and

$$
\mathcal{C}=5^{D} 4^{\eta+1} d \mu_{d}\left(g \mu_{g} e^{\lambda(b)}\right)^{\eta+D},
$$

and we denote $\overline{\Gamma(z)}=\left(\gamma_{1} z, \ldots, \gamma_{D} z\right)$ for brevity.

Proof. Firstly, we let $w \mid \infty$ and we estimate the sum

$$
\begin{aligned}
& \left|\sum_{j=0}^{\rho-l-i-k}\left(\begin{array}{c}
\rho-l-i-k \\
j
\end{array}\right) \frac{(-1)^{j}}{B+k+j}\right|_{w} \\
& \quad \leq \max _{0 \leq j \leq \rho-l-i-k}\left|\frac{1}{B+k+j}\right| \sum_{j=0}^{\rho-l-i-k}\left(\begin{array}{c}
\rho-l-i-k \\
j
\end{array}\right) \leq M 2^{\rho-l-i-k}
\end{aligned}
$$

where

$$
M:=\max _{0 \leq j \leq \rho-l-i-k}\left|\frac{1}{B+k+j}\right| \quad \text { for } l \text { large enough. }
$$

On the other hand, another simple application of the binomial theorem yields

$$
\left|\left(\begin{array}{c}
C+k+l-1 \\
l
\end{array}\right)\left(\begin{array}{c}
C+\rho-i-1 \\
\rho-l-i-k
\end{array}\right)\right| \leq 2^{2\lceil|C|\rceil+\rho+l+k-i-2},
$$

and therefore by $(5.2)$ and $(5.3)$ we obtain

$$
\begin{aligned}
\left.\mid \Omega_{l, \lambda}^{(D)}(a, b, d) / l !\right)\left.A_{l, \lambda}(z)\right|_{w} \\
\quad=\left|\Omega_{l, \lambda}^{(D)}(a, b, d) \sum_{i=0}^{D l} \sigma_{D l-i, l} \frac{(C)_{\rho-i}}{l !(B)_{D l+\lambda-i}} z^{i}\right|_{w} \\
\quad \leq \Omega_{l, \lambda}^{(D)}(a, b, d) \sum_{i=0}^{D l}\left|\sigma_{D l-i, l}\right| w\left|\frac{(C)_{\rho-i}}{l !(B)_{D l+\lambda-i}}\right||z|_{w}^{i} \\
\quad \leq \Omega_{l, \lambda}^{(D)}(a, b, d) M 4^{\lceil|C|\rceil+\rho-1} \sum_{i=0}^{D l}\left|\sigma_{D l-i, l}\right|_{w}\left|\frac{z}{4}\right|_{w}^{i}
\end{aligned}
$$




$$
\begin{aligned}
& =M \Omega_{l, \lambda}^{(D)}(a, b, d) 4^{\lceil|C|\rceil+\rho-1-D l}|z|_{w}^{D l} \sum_{i=0}^{D l}\left|\sigma_{i, l}\right|_{w}\left|\frac{4}{z}\right|_{w}^{i} \\
& \leq M \Omega_{l, \lambda}^{(D)}(a, b, d) 4^{\lceil|C|\rceil+\lambda+l-2}|z|_{w}^{D l} \prod_{t=1}^{D}\left(\left|\gamma_{t}\right|_{w}+4 /|z|_{w}\right)^{l} \\
& =M \Omega_{l, \lambda}^{(D)}(a, b, d) 4^{\lceil|C|\rceil+\lambda+l-2} \prod_{t=1}^{D}\left(\left|\gamma_{t} z\right|_{w}+4\right)^{l} \\
& \leq M \Omega_{l, \lambda}^{(D)}(a, b, d) 5^{D l} 4^{\lceil|C|\rceil+\lambda+l-2} \prod_{t=1}^{D}\left|\gamma_{t} z\right|_{w}^{*} .
\end{aligned}
$$

Similarly,

$$
\begin{aligned}
\mid \Omega_{l, \lambda}^{(D)} & (a, b, d) / l !)\left.B_{l, \lambda}^{(\nu)}(z)\right|_{w} \\
& =\Omega_{l, \lambda}^{(D)}(a, b, d)\left|\sum_{n=0}^{\lambda+D l-1} z^{n} \sum_{i+k=n} \sigma_{D l-i, l}\left(a_{i} f_{k} / l !\right) \gamma_{\nu}^{n-i}\right|_{w} \\
& \leq \Omega_{l, \lambda}^{(D)}(a, b, d) \sum_{n=0}^{\lambda+D l-1}\left|\gamma_{\nu} z\right|_{w}^{n} \sum_{i+k=n}\left|\sigma_{D l-i, l}\right|_{w}\left|\left(a_{i} f_{k} / l !\right)\right|\left|\gamma_{\nu}\right|_{w}^{-i} \\
& \leq\left. M \Omega_{l, \lambda}^{(D)}(a, b, d) \sum_{n=0}^{\lambda+D l-1}\left|\gamma_{\nu} z\right|_{w}^{n}\left|\gamma_{\nu}\right|_{w}^{-D l} 4^{\lceil|C|\rceil+\lambda+l-2} \sum_{i=0}^{D l}\left|\sigma_{i, l}\right|\right|_{w}\left|4 \gamma_{\nu}\right|_{w}^{i} \\
& \leq M \Omega_{l, \lambda}^{(D)}(a, b, d) \sum_{n=0}^{\lambda+D l-1}\left|\gamma_{\nu} z\right|_{w}^{n}\left|\gamma_{\nu}\right|_{w}^{-D l} 4^{\lceil|C|\rceil+\lambda+l-2} \prod_{t=1}^{D}\left(\left|\gamma_{t}\right|_{w}+4\left|\gamma_{\nu}\right|_{w}\right)^{l} \\
& \leq M \Omega_{l, \lambda}^{(D)}(a, b, d) 4^{\lceil|C|\rceil+\lambda+l-2} \sum_{n=0}^{\lambda+D l-1}\left|\gamma_{\nu} z\right|_{w}^{n-D l} \prod_{t=1}^{D}\left(\left|z \gamma_{t}\right|_{w}+4\left|z \gamma_{\nu}\right|_{w}\right)^{l} \\
& \leq M \Omega_{l, \lambda}^{(D)}(a, b, d) 5^{D l}{ }_{4}^{\lceil|C|\rceil+\lambda+l-2}(D l+\lambda)\left(|\overline{\Gamma(z)}|_{w}^{*}\right)^{D l+\lambda-1} .
\end{aligned}
$$

If $w \mid p$, then by Lemma 5.2,

$$
\begin{aligned}
& \left|\left(\Omega_{l, \lambda}^{(D)}(a, b, d) / l !\right) B_{l, \lambda}^{(\nu)}(z)\right|_{w} \\
& \quad=\max _{0 \leq n \leq D l+\lambda-1}\left|z^{n} \sum_{\substack{i+k=n \\
0 \leq i \leq D l}} \sigma_{D l-i, l} \gamma_{\nu}^{k}\left(\Omega_{l, \lambda}^{(D)}(a, b, d) / l !\right) a_{i} f_{k}\right|_{w} \\
& \quad \leq \max _{0 \leq n \leq D l+\lambda-1}|z|_{w}^{n} \max _{\substack{i+k=n \\
0 \leq i \leq D l}}|\bar{\Gamma}|_{w}^{k}\left|\sigma_{D l-i, l}\right|_{w} \\
& \quad \leq\left.\max _{0 \leq n \leq D l+\lambda-1}\left(|z|_{w}|\bar{\Gamma}|_{w}\right)^{n} \max _{\substack{i+k=n \\
0 \leq i \leq D l}} \max _{\substack{i_{1}+\cdots+i_{D}=D l-i \\
0.1}}\left|\gamma_{1}\right|_{w}^{l-i_{1}} \ldots\left|\gamma_{D}\right|\right|_{w} ^{l-i_{D}}|\bar{\Gamma}|_{w}^{-i}
\end{aligned}
$$




$$
\begin{aligned}
= & \max _{0 \leq n \leq D l+\lambda-1}|\overline{\Gamma(z)}|_{w}^{n} \\
& \times \max _{\substack{i+k=n \\
0 \leq i \leq D l}} \max _{i_{1}+\cdots+i_{D}=D l-i}\left(\left|\gamma_{1}\right|_{w} /|\bar{\Gamma}|_{w}\right)^{l-i_{1}} \cdots\left(\left|\gamma_{D}\right|_{w} /|\bar{\Gamma}|_{w}\right)^{l-i_{D}} \\
\leq & \left(|\overline{\Gamma(z)}|_{w}^{*}\right)^{D l+\lambda-1},
\end{aligned}
$$

and also

$$
\begin{aligned}
\left|\left(\Omega_{l, \lambda}^{(D)}(a, b, d) / l !\right) A_{l, \lambda}(z)\right|_{w} & \leq \max _{i_{1}+\cdots+i_{D}=D l-i}\left|\gamma_{1}\right|_{w}^{l-i_{1}} \cdots\left|\gamma_{D}\right|_{w}^{l-i_{D}}|z|_{w}^{i} \\
& \leq\left(|\overline{\Gamma(z)}|_{w}^{*}\right)^{D l} .
\end{aligned}
$$

Thus, for every $w \in \mathcal{M}$ we are left with the upper bound

$$
\begin{aligned}
\max \left\{\left|\frac{\Omega_{l, \lambda}^{(D)}(a, b, d)}{l !} A_{l, \lambda}(z)\right|_{w},\left|\frac{\Omega_{l, \lambda}^{(D)}(a, b, d)}{l !} B_{l, \lambda}^{(\nu)}(z)\right|_{w}\right\} & \leq \mathcal{C}_{l}^{\delta_{w}}\left(\left.\overline{\Gamma(z)}\right|_{w} ^{*}\right)^{D l+\lambda}
\end{aligned}
$$

where

$$
\mathcal{C}_{l}=M \Omega_{l, \lambda}^{(D)}(a, b, d)(D l+\lambda) 5^{D l} 4^{\lceil|C|\rceil+\lambda+l-2},
$$

and $\delta_{w}=1$ if $w \mid \infty$, while $\delta_{w}=0$ if $w \mid p$.

By setting $\lambda=\lfloor\eta l\rfloor, \eta \geq 0$, and by making use of the well-known estimates (the first inequality follows directly from 2.1) and we refer to Lemma 1 of [1] for the asymptotic bound)

$$
\omega_{N}(d) \leq \mu_{d}^{N}, \quad \lim _{M \rightarrow \infty} \frac{\ln \left(E_{M}(a, b)\right)}{M}=\frac{b}{\phi(b)} \sum_{\substack{j=1 \\ \operatorname{gcd}(j, b)=1}}^{b} \frac{1}{j}=\lambda(b),
$$

we are led to the estimate

$$
\begin{aligned}
\Omega_{l, \lambda}^{(D)}(a, b, d) & =d^{l-2} g^{D l+\lambda} \omega_{\rho-l}(g) \omega_{l}(d) E_{D l+\lambda}(a, b) \\
& \leq c_{0}\left(g \mu_{g} e^{\lambda(b)}\right)^{(\eta+D) l}\left(d \mu_{d}\right)^{l}
\end{aligned}
$$

with $c_{0}>0$. From the upper bound 5.6 we obtain in turn

$$
\begin{aligned}
\max \{\mid & \left.\left.\left(\Omega_{l, \lambda}^{(D)}(a, b, d) / l !\right) A_{l, \lambda}(z)\right|_{w},\left|\left(\Omega_{l, \lambda}^{(D)}(a, b, d) / l !\right) B_{l, \lambda}^{(\nu)}(z)\right|_{w}\right\} \\
\leq & c_{0}(D l+\lambda) \mathcal{C}^{\delta_{w} l}\left(|\overline{\Gamma(z)}|_{w}^{*}\right)^{(D+\eta) l} \leq \mathcal{C}^{\delta_{w} l}\left(|\overline{\Gamma(z)}|_{w}^{*}\right)^{(D+\eta) l+c_{1} \log l},
\end{aligned}
$$

where $c_{1}>0$ and $\mathcal{C}=5^{D} 4^{\eta+1} d \mu_{d}\left(g \mu_{g} e^{\lambda(b)}\right)^{\eta+D}$, proving the lemma.

LEMMA 5.4. Let $|\overline{\Gamma(z)}|_{v}<1$ and $\operatorname{ord}_{p}(d) \geq \operatorname{ord}_{p}(b)$ if $v \mid p$. Then

$$
\left|\left(\Omega_{l, \lambda}^{(D)}(a, b, d) / l !\right) R_{l, \lambda}^{(\nu)}(z)\right|_{v} \leq \mathcal{C}_{r}^{\delta_{v} l}|\overline{\Gamma(z)}|_{v}^{(D+\eta+1) l-c_{3} \log l}, \quad c_{3}>0,
$$

for every $v \in \mathcal{M}$, where

$$
\mathcal{C}_{r}=d \mu_{d}\left(g \mu_{g} e^{\lambda(b)}\right)^{\eta+D} 2^{\eta+1} 3^{D} .
$$


Proof. Let $v \mid \infty$. Then

$$
\left|R_{l, \lambda}^{(\nu)}(z)\right|_{v} \leq|z|_{v}^{\rho+1} \sum_{k=0}^{\infty}\left|g_{l, \lambda, \rho+k+1}^{(\nu)}\right| v|z|_{v}^{k}
$$

where, for sufficiently large $l$,

$$
\begin{aligned}
&\left|g_{l, \lambda, \rho+k+1}^{(\nu)}\right|_{v}=\left|\gamma_{\nu}\right|_{v}^{\rho+k+1}\left|\sum_{i=0}^{D l} \sigma_{D l-i, l} \frac{(C)_{\rho-i}(B)_{\rho+k+1-i}}{(B)_{D l-i+\lambda}(C)_{\rho+k+1-i}} \gamma_{\nu}^{-i}\right|_{v} \\
&=\left|\gamma_{\nu}\right|_{v}^{l+\lambda+k}\left|\sum_{i=0}^{D l} \sigma_{i, l}(B+\lambda+i)_{l-1} \frac{(B+\rho+i-D l)_{k+1}}{(C+\rho+i-D l)_{k+1}} \gamma_{\nu}^{i}\right|_{v} \\
&=(l-1) ! \cdot\left|\gamma_{\nu}\right|_{v}^{l+\lambda+k} \\
& \quad \times\left|\sum_{i=0}^{D l} \sigma_{i, l}\left(\begin{array}{c}
B+\lambda+i+l-2 \\
\leq
\end{array}\right) \prod_{j=0}^{k}\left(1+\frac{B-C}{C+\rho-D l+i+j}\right) \gamma_{\nu}^{i}\right|_{v} \\
& \leq(l-1) ! \cdot\left|\gamma_{\nu}\right|_{v}^{l+\lambda+k} 2^{\lceil|B|\rceil+\lambda+l-2} \\
& \times \sum_{i=0}^{D l}\left|\sigma_{i, l}\left(2 \gamma_{\nu}\right)^{i}\right|_{v} \prod_{j=0}^{k}\left|1+\frac{B-C}{C+\rho-D l+i+j}\right| \\
& \leq(l-1) ! \cdot\left|\gamma_{\nu}\right|_{v}^{l+\lambda+k} 2^{\lceil|B|\rceil+\lambda+l-2}\left(1+\frac{|B-C|}{|C+l-1|}\right)^{k+1} \sum_{i=0}^{D l}\left|\sigma_{i, l}\left(2 \gamma_{\nu}\right)^{i}\right|_{v} \\
&=(l-1) ! \cdot\left|\gamma_{\nu}\right|_{v}^{l+\lambda+k} 2^{\lceil|B|\rceil+\lambda+l-2}\left(1+\frac{|B-C|}{|C+l-1|}\right)^{k+1} \prod_{t=1}^{D}\left(\left|\gamma_{t}\right|_{v}+2\left|\gamma_{\nu}\right|_{v}\right)^{l} \\
& \leq(l-1) ! \cdot|\bar{\Gamma}|_{v}^{\rho+1} 2^{\lceil|B|\rceil+\lambda+l-2} 3^{D l} \\
& \times\left(1+\frac{|B-C|}{|C+l-1|}\right)\left(|\bar{\Gamma}|_{v}\left(1+\frac{|B-C|}{|C+l-1|}\right)\right)^{k} \cdot
\end{aligned}
$$

Thus, we arrive at the estimate

$$
\begin{aligned}
\left|R_{l, \lambda}^{(\nu)}(z)\right|_{v} \leq & |\overline{\Gamma(z)}|_{v}^{\rho+1}(l-1) ! 2^{\lceil|B|\rceil+\lambda+l-2} 3^{D l}\left(1+\frac{|B-C|}{|C+l-1|}\right) \\
& \times \sum_{k=0}^{\infty}\left(|\bar{\Gamma}|_{v}\left(1+\frac{|B-C|}{|C+l-1|}\right)\right)^{k}|z|_{v}^{k} \\
\leq & c_{2}(l-1) ! 2^{\lambda+l} 3^{D l}|\overline{\Gamma(z)}|_{v}^{\rho+1}, \quad c_{2}=c_{2}(z)>0,
\end{aligned}
$$

since

$$
\frac{|B-C|}{|C+l-1|} \rightarrow 0 \quad \text { and } \quad|\overline{\Gamma(z)}|_{v}<1
$$


By this upper bound we obtain

$$
\left|\left(\Omega_{l, \lambda}^{(D)}(a, b, d) / l !\right) R_{l, \lambda}^{(\nu)}(z)\right|_{v} \leq \frac{c_{2}}{l}\left(d \mu_{d}\left(g \mu_{g} e^{\lambda(b)}\right)^{D+\eta} 2^{\eta+1} 3^{D}|\overline{\Gamma(z)}|_{v}^{D+\eta+1}\right)^{l}
$$

for $v \mid \infty$.

Let now $v \mid p$. Then

$$
\left(\Omega_{l, \lambda}^{(D)}(a, b, d) / l !\right) R_{l, \lambda}^{(\nu)}(z)=\left(\Omega_{l, \lambda}^{(D)}(a, b, d) / l !\right) z^{\rho+1} \sum_{k=0}^{\infty} g_{l, \lambda, \rho+k+1}^{(\nu)} z^{k},
$$

where

$$
g_{l, \lambda, \rho+k+1}^{(\nu)}=\sum_{i=0}^{D l} \sigma_{D l-i} a_{i} f_{\rho+k+1-i} \gamma_{\nu}^{\rho+k+1-i}, \quad k=0,1, \ldots
$$

By Lemma 5.2,

$$
\begin{aligned}
\mid\left(\Omega_{l, \lambda}^{(D)}(a, b, d) / l !\right) & \left.g_{l, \lambda, \rho+k+1}^{(\nu)}\right|_{v} \\
& \leq \max _{0 \leq i \leq D l}\left|\left(\Omega_{l, \lambda}^{(D)}(a, b, d) / l !\right) \sigma_{D l-i, l} a_{i} f_{\rho+k+1-i} \gamma_{\nu}^{\rho+k+1-i}\right|_{v} \\
& \leq \max _{0 \leq i \leq D l}\left|\sigma_{D l-i, l}\right|_{v}|\bar{\Gamma}|_{v}^{-i}\left|f_{\rho+k+1-i}\right|_{v}|\bar{\Gamma}|_{v}^{\rho+k+1} \\
& \leq|\bar{\Gamma}|_{v}^{\rho+k+1} \max _{0 \leq i \leq D l}\left|f_{\rho+k+1-i}\right|_{v},
\end{aligned}
$$

with

$$
f_{\rho+k+1-i}=\left(\frac{d}{b}\right)^{\rho+k+1-i} \frac{a(a+b) \cdots(a+b(\rho+k-i))}{c(c+d) \cdots(c+d(\rho+k-i))} .
$$

We assume that $\operatorname{ord}_{p}(d) \geq \operatorname{ord}_{p}(b)$. Firstly, if $p \mid b d$, then $\left|f_{\rho+k+1-i}\right|_{p} \leq 1$, and hence we may suppose $p \nmid b d$. If $p^{m_{p}(x, y)} \mid(x ; y)_{\rho+k+1-i}, x, y \in \mathbb{Z}, y>0$, $m_{p}(x, y)$ being maximal, then

$$
\operatorname{ord}_{p}((\rho+k+1-i) !) \leq m_{p}(x, y) \leq \operatorname{ord}_{p}((\rho+k+1-i) !)+M_{p}(x, y)
$$

by Lemma 5.1, where $M_{p}(x, y)=\lfloor\log (|x|+y(\rho+k-i)) / \log p\rfloor$. This yields

$$
\left|f_{\rho+k+1-i}\right|_{p}=p^{m_{p}(c, d)-m_{p}(a, b)} \leq p^{M_{p}(c, d)} \leq d(|C|+\rho+k)
$$

for every $0 \leq i \leq D l$, due to the inequality

$$
M_{p}(c, d) \leq \frac{\log (d(|C|+\rho+k))}{\log p}, \quad k=0,1,2, \ldots
$$

By the above estimates, for $l$ large enough $\left(|\overline{\Gamma(z)}|_{v}<1\right)$ we get

$$
\begin{aligned}
& \left|\left(\Omega_{l, \lambda}^{(D)}(a, b, d) / l !\right) R_{l, \lambda}^{(\nu)}(z)\right|_{v} \leq|\overline{\Gamma(z)}|_{v}^{\rho+1} \max _{k \geq 0}\left|f_{\rho+k+1-i}\right|_{p}|\overline{\Gamma(z)}|_{v}^{k} \\
& \leq d(|C|+2 \rho)|\overline{\Gamma(z)}|_{v}^{\rho+1} \\
& \leq 2(|C|+d)(D+\eta+1) l|\overline{\Gamma(z)}|_{v}^{(D+\eta+1) l} \\
& \leq|\overline{\Gamma(z)}|_{v}^{(D+\eta+1) l-c_{3} \log l}, \quad c_{3}>0 \text {. }
\end{aligned}
$$


REMARK 5.5. The estimates of the polynomials may, in many special cases of $B$ and $C$, be sharpened. For example, in the case of the logarithmic function, i.e. $B=1, C=2$, we have

$$
\begin{aligned}
& A_{l, \lambda}(z)=\sum_{i=0}^{D l} \sigma_{i, l}\left(\begin{array}{c}
l+\lambda+i \\
l
\end{array}\right) z^{D l-i}, \\
& B_{l, \lambda}^{(\nu)}(z)=\sum_{n=0}^{D l+\lambda-1} z^{n} \sum_{i+k=n} \sigma_{D l-i, l} \frac{\left(\begin{array}{c}
l+\lambda+D l-i \\
l
\end{array}\right)}{k+1} \gamma_{\nu}^{k}, \\
& R_{l, \lambda}^{(\nu)}(z)=\left(z \gamma_{\nu}\right)^{\rho+1} \sum_{n=0}^{\infty} \sum_{i=0}^{D l} \sigma_{D l-i, l} \frac{\left(\begin{array}{c}
l+\lambda+D l-i \\
l
\end{array}\right)}{\rho+n-i+2} \gamma_{\nu}^{n-i} .
\end{aligned}
$$

This yields $\mathcal{C}=\mathcal{C}_{r}=3^{D} 2^{\eta+1} e^{\eta+D}$ in Lemma 5.3. The polynomial 5.9 is the same as the denominator polynomial $P_{0, n, m}(z)$ appearing in [28] with $r=D, l=n$ and $m=\lambda$.

6. Proof of Theorem 2.1 and the corollaries. Let

$$
\begin{aligned}
a_{l, \lambda} & :=\left(\Omega_{l, \lambda}^{(D)}(a, b, d) / l !\right) A_{l, \lambda}(1) \in \mathbb{Z}\left[\gamma_{1}, \ldots, \gamma_{D}\right], \\
b_{l, \lambda}^{(\nu)} & :=\left(\Omega_{l, \lambda}^{(D)}(a, b, d) / l !\right) B_{l, \lambda}^{(\nu)}(1) \in \mathbb{Z}\left[\gamma_{1}, \ldots, \gamma_{D}\right], \\
r_{l, \lambda}^{(\nu)} & :=\left(\Omega_{l, \lambda}^{(D)}(a, b, d) / l !\right) R_{l, \lambda}^{(\nu)}(1),
\end{aligned}
$$

where by Lemmas 5.3 and 5.4

$\max \left\{\left\|a_{l, \lambda}\right\|_{w},\left\|b_{l, \lambda}^{(\nu)}\right\|_{w}\right\} \leq \mathcal{C}^{\left(\kappa_{w} / \kappa\right) \delta_{w} l}\left(\|\bar{\Gamma}\|_{w}^{*}\right)^{(D+\eta) l+\mathcal{O}(\log l)}=: \mathcal{P}_{w}(l) \quad \forall w \in \mathcal{M}$ and

$$
\left\|r_{l, \lambda}^{(\nu)}\right\|_{v} \leq \mathcal{C}_{r}^{\left(\kappa_{v} / \kappa\right) \delta_{v} l}\|\bar{\Gamma}\|_{v}^{(D+\eta+1) l-\mathcal{O}(\log l)}=: \mathcal{R}_{v}(l) .
$$

Furthermore,

$$
\prod_{w \in \mathcal{M}} \mathcal{P}_{w}(l)=\mathcal{C}^{l} H(\bar{\Gamma})^{(D+\eta) l+\mathcal{O}(\log l)}=: \mathcal{P}(l)
$$

and suppose

$$
\|\bar{\Gamma}\|_{v}^{D+\eta+1} \mathcal{C} H(\bar{\Gamma})^{D+\eta}<\left(\frac{\mathcal{C}}{\mathcal{C}_{r}}\right)^{\kappa_{v} \delta_{v} / \kappa}=: \mathcal{E}_{v}
$$

i.e.

$$
\log \|\bar{\Gamma}\|_{v}^{-1}>\frac{\log \mathcal{C}+(D+\eta) \log H(\bar{\Gamma})-\log \mathcal{E}_{v}}{D+\eta+1} .
$$

Since for all $\varepsilon>0$ there exists $l_{0} \in \mathbb{N}$ such that

$$
\|\bar{\Gamma}\|_{v}^{-\mathcal{O}(\log l)} \leq\|\bar{\Gamma}\|_{v}^{-\varepsilon l} \quad \forall l \geq l_{0}
$$


and

$$
H(\bar{\Gamma})^{\mathcal{O}(\log l)} \leq H(\bar{\Gamma})^{\varepsilon l} \quad \forall l \geq l_{0},
$$

for every $l \geq l_{0}$ we have

$$
\mathcal{P}(l) \leq \mathcal{C}^{l} H(\bar{\Gamma})^{(D+\eta+\varepsilon) l}, \quad \mathcal{R}_{v}(l) \leq \mathcal{C}_{r}^{\left(\kappa_{v} / \kappa\right) \delta_{v} l}\|\bar{\Gamma}\|_{v}^{(D+\eta+1-\varepsilon) l} .
$$

Next, consider the linear form

$$
L=b_{0}+\sum_{\nu=1}^{D} b_{\nu} g_{\nu}(1), \quad \bar{B}=\left(b_{0}, \ldots, b_{D}\right) \in \mathbb{K}^{D+1} \backslash\{\overline{0}\} .
$$

Multiplication of this form by $a_{l, \lambda_{0}}$ yields

$$
a_{l, \lambda_{0}} L=\tilde{L}+\sum_{\nu=1}^{D} b_{\nu} r_{l, \lambda_{0}}^{(\nu)}
$$

where

$$
\tilde{L}=b_{0} a_{l, \lambda_{0}}+\sum_{\nu=1}^{D} b_{\nu} b_{l, \lambda_{0}}^{(\nu)} \in \mathbb{K}^{*}
$$

for some $\lambda_{0}=\lambda+\tau$, where $\tau \in[0, D-1]$, by the nonvanishing of the determinant in Theorem 4.3. By the product formula we have

$$
\begin{aligned}
1= & \prod_{w \in \mathcal{M}}\|\tilde{L}\|_{w}=\prod_{\substack{w \in \mathcal{M} \\
w \neq v}}\left\|b_{0} a_{l, \lambda_{0}}+\sum_{\nu=1}^{D} b_{\nu} b_{l, \lambda_{0}}^{(\nu)}\right\|_{w}\left\|a_{l, \lambda_{0}} L-\sum_{\nu=1}^{D} b_{\nu} r_{l, \lambda_{0}}^{(\nu)}\right\|_{v} \\
\leq & \prod_{\substack{w \in \mathcal{M} \\
w \neq v}}\|\bar{B}\|_{w}^{*} \mathcal{P}_{w}(l)\left\{\mathcal{P}_{v}(l)\|L\|_{v}+\|\bar{B}\|_{v}^{*} \mathcal{R}_{v}(l)\right\} \\
= & \mathcal{P}(l)\|\bar{B}\|_{v}^{*-1} H(\bar{B})\|L\|_{v}+H(\bar{B}) \mathcal{C}^{-\left(\kappa_{v} / \kappa\right) \delta_{v} l} \mathcal{P}(l) \mathcal{R}_{v}(l) \\
\leq & \mathcal{C}^{l} H(\bar{\Gamma})^{(D+\eta+\varepsilon) l}\|\bar{B}\|_{v}^{*-1} H(\bar{B})\|L\|_{v} \\
& +\frac{1}{2} H(\bar{B})\left(\mathcal{C} H(\bar{\Gamma})^{D+\eta+\varepsilon} \mathcal{E}_{v}^{-1}\|\bar{\Gamma}\|_{v}^{D+\eta+1-\varepsilon}\right)^{l} .
\end{aligned}
$$

Since $\varepsilon>0$ may be chosen arbitrarily small, we know from 6.3 that

$$
\left(\mathcal{C} H(\bar{\Gamma})^{D+\eta+\varepsilon} \mathcal{E}_{v}^{-1}\|\bar{\Gamma}\|_{v}^{D+\eta+1-\varepsilon}\right)^{l} \rightarrow 0 \quad \text { as } l \rightarrow \infty,
$$

and therefore we may find, by choosing $H(\bar{B})$ large enough, say $H(\bar{B}) \geq H_{0}$, the maximum $l_{1}$ of $l \geq l_{0}$ such that

$$
\frac{1}{2} H(\bar{B})\left(\mathcal{C} H(\bar{\Gamma})^{D+\eta} \mathcal{E}_{v}^{-1}\|\bar{\Gamma}\|_{v}^{D+\eta+1}\right)^{l_{1}} \geq \frac{1}{2} .
$$

This in turn implies, by taking the logarithm on both sides of (6.4), that

$$
\log H(\bar{B})+l_{1} \log \left(\mathcal{E}_{v}^{-1}\|\bar{\Gamma}\|_{v}^{D+\eta+1} \mathcal{C} H(\bar{\Gamma})^{D+\eta}\right) \geq 0
$$


and

$$
l_{1} \leq-\frac{\log H(\bar{B})}{\log \left(\mathcal{E}_{v}^{-1}\|\bar{\Gamma}\|_{v}^{D+\eta+1} \mathcal{C} H(\bar{\Gamma})^{D+\eta}\right)} .
$$

Thus, for $l=l_{1}+1$,

$$
\mathcal{C}^{l_{1}+1} H(\bar{\Gamma})^{(D+\eta)\left(l_{1}+1\right)}\|\bar{B}\|_{v}^{*-1} H(\bar{B})\|L\|_{v} \geq \frac{1}{2}
$$

by the product formula, and

$$
\begin{aligned}
\|L\|_{v} & \geq \frac{\|\bar{B}\|_{v}^{*}}{2 H(\bar{B})\left(\mathcal{C} H(\bar{\Gamma})^{D+\eta}\right)^{l_{1}+1}} \\
& \geq \frac{C_{1}\|\bar{B}\|_{v}^{*}}{H(\bar{B})^{1-\log \left(\mathcal{C} H(\bar{\Gamma})^{D+\eta}\right) / \log \left(\mathcal{E}_{v}^{-1}\|\bar{\Gamma}\|_{v}^{D+\eta+1} \mathcal{C} H(\bar{\Gamma})^{D+\eta}\right)}}, \quad C_{1}>0 .
\end{aligned}
$$

Finally,

$$
|L|_{v} \geq C|\bar{B}|^{*} H(\bar{B})^{-\mu}, \quad C>0
$$

where

$$
\begin{aligned}
\mu & =\frac{\kappa}{\kappa_{v}} \cdot \frac{\log \left(\mathcal{E}_{v}^{-1}\|\bar{\Gamma}\|_{v}^{D+\eta+1}\right)}{\log \left(\mathcal{E}_{v}^{-1}\|\bar{\Gamma}\|_{v}^{D+\eta+1} \mathcal{C} H(\bar{\Gamma})^{D+\eta}\right)} \\
& =\frac{\kappa(D+\eta+1) \log |\bar{\Gamma}|_{v}-\kappa \delta_{v} \log \left((5 / 3)^{D} 2^{\eta+1}\right)}{\left(\kappa-\kappa_{v} \delta_{v}\right) \log \mathcal{C}+\kappa(D+\eta) \log H(\bar{\Gamma})+\kappa_{v}(D+\eta+1) \log |\bar{\Gamma}|_{v}+\kappa_{v} \delta_{v} \log \mathcal{C}_{r}}
\end{aligned}
$$

and

$$
\mathcal{C}=5^{D} 4^{\eta+1} d \mu_{d}\left(g \mu_{g} e^{\lambda(b)}\right)^{D+\eta}, \quad \mathcal{C}_{r}=3^{D} 2^{\eta+1} d \mu_{d}\left(g \mu_{g} e^{\lambda(b)}\right)^{D+\eta} .
$$

This proves Theorem 2.1.

Proof of the corollaries. If $\mathbb{K}=\mathbb{Q}$ or $\mathbb{I}$ and $\bar{B} \in \mathbb{Z}_{\mathbb{K}}^{D+1} \backslash\{\overline{0}\}$, then $H(\bar{B})=$ $\max \left\{\left|b_{0}\right|, \ldots,\left|b_{D}\right|\right\}$ and

$$
|L|_{v} \geq C H(\bar{B})^{-\mu+1}, \quad C>0,
$$

by the lower bound 6.5 . Set $\gamma_{\nu}=\xi_{\nu} / q$, where $\xi_{\nu} \in \mathbb{I}^{*}, q \in \mathbb{Z}_{\mathbb{I}} \backslash\{0\}$ and write $\bar{\Xi}=\left(\xi_{1}, \ldots, \xi_{D}\right)$. Since $|\bar{\Gamma}|=|q|^{-1}|\bar{\Xi}|<1$, we have

$$
H(\bar{\Gamma}) \leq \frac{H(\bar{\Xi}) H\left(q^{-1}\right)}{\max \{1,|\bar{\Xi}|\}}=\frac{H(\bar{\Xi}) H(q)}{|\bar{\Xi}|^{*}}=H(\bar{\Xi})|\bar{\Xi}|^{*-1}|q|,
$$

and the claim follows from 6.3. The remaining results in Corollary 2.2 are straightforward computations, and Corollary 2.3 is a direct consequence of Theorem 2.1.

Acknowledgements. The author would like to thank the anonymous referee for the useful comments which improved the presentation of this article. 


\section{References}

[1] K. Alladi and M. L. Robinson, Legendre polynomials and irrationality, J. Reine Angew. Math. 318 (1980), 137-155.

[2] A. Baker, Rational approximations to $\sqrt[3]{2}$ and other algebraic numbers, Quart. J. Math. Oxford Ser. (2) 15 (1964), 375-383.

[3] M. A. Bennett, Simultaneous rational approximation to binomial functions, Trans. Amer. Math. Soc. 348 (1996), 1717-1738.

[4] F. Beukers, T. Matala-aho and K. Väänänen, Remarks on the arithmetic properties of the values of hypergeometric functions, Acta Arith. 42 (1983), 281-289.

[5] E. Bombieri, On G-functions, in: Recent Progress in Analytic Number Theory, Vol. 2 (Durham, 1979), Academic Press, London, 1981, 1-67.

[6] P. Bundschuh, Zur Approximation gewisser p-adischer algebraischer Zahlen durch rationalen Zahlen, J. Reine Angew. Math. 265 (1974), 154-159.

[7] G. V. Chudnovsky, Padé approximations to the generalized hypergeometric functions, J. Math. Pures Appl. (9) 58 (1979), 445-476.

[8] —, On the method of Thue-Siegel, Ann. of Math. (2) 117 (1983), 325-382.

[9] - On applications of diophantine approximations, Proc. Nat. Acad. Sci. U.S.A. Phys. Sci. 81 (1984), 7261-7265.

[10] D. V. Chudnovsky and G. V. Chudnovsky, Applications of Padé approximations to Diophantine inequalities in values of G-functions, in: Lecture Notes in Math. 1135, Springer, 1985, 9-51.

[11] R. P. Flowe and G. A. Harris, A note on generalized Vandermonde determinants, SIAM J. Matrix Anal. Appl. 14 (1993), 1146-1151.

[12] A. I. Galochkin, Lower estimates for linear forms of $G$-functions values, Moscow Univ. Math. Bull. 51 (1996), 18-22.

[13] M. Hata, Legendre type polynomials and irrationality measures, J. Reine Angew. Math. 407 (1990), 335-347.

[14] - The irrationality of $\log (1+1 / q) \log (1-1 / q)$, Trans. Amer. Math. Soc. 350 (1998), 2311-2327.

[15] A. Heimonen, T. Matala-aho and K. Väänänen, On irrationality measures of the values of Gauss hypergeometric function, Manuscripta Math. 81 (1993), 183-202.

[16] P. L. Ivankov, Arithmetic properties of values of hypergeometric functions, Mat. Sb. 182 (1991), 283-302.

[17] C. Krattenthaler, Advanced determinant calculus, in: The Andrews Festschrift (Maratea, 1998), Sem. Lothar. Combin. 42 (1999), Art. B42q, 67 pp. (electronic).

[18] K. Mahler, Ein Beweis des Thue-Siegelschen Satzes über die Approximation algebraischer Zahlen für binomische Gleichungen, Math. Ann. 105 (1931), 267-276.

[19] W. Maier, Potenzreihen irrationalen Grenzwertes, J. Reine Angew. Math. 156 (1927), 93-148.

[20] T. Matala-aho, Type II Padé approximations of generalized hypergeometric series, submitted (2009).

[21] C. Méray, Sur un déterminant dont celui de Vandermonde n'est qu'un cas particulier, Revue de Mathématiques Spéciales 9 (1899), 217-219.

[22] V. Merilä, On arithmetical properties of certain q-series, Results Math. 53 (2009), $129-151$.

[23] E. M. Nikishin, A system of Markov functions, Vestnik Moskov. Univ. Ser. I Mat. Mekh. 1979, no. 4, 60-63; English transl.: Moscow Univ. Math. Bull. 34 (1979), no. $4,63-66$. 
[24] E. M. Nikishin, Irrationality of values of functions $F(x, s)$, Mat. Sb. 109 (151) (1979), 410-417; English transl.: Math. USSR-Sb. 37 (1980), 381-388.

[25] —, Simultaneous Padé approximants, Mat. Sb. 113 (155) (1980), 499-519; English transl.: Math. USSR-Sb. 41 (1982), 409-425.

[26] —, A set of power series, Sibirsk. Mat. Zh. 22 (1981), no. 4, 164-168; English transl.: Siberian Math. J. 22 (1981), 611-615.

[27] - Arithmetic properties of the Markov function for the Jacobi weight, Anal. Math. 8 (1982), 39-46.

[28] G. Rhin et P. Toffin, Approximants de Padé simultanés de logarithmes, J. Number Theory 24 (1986), 282-297.

[29] E. A. Rukhadze, Lower estimate for rational approximations of $\ln 2$, Vestnik Moskov. Univ. Ser. I Mat. Mekh. 1987, no. 6, 25-29; English transl.: Moscow Univ. Math. Bull. 42 (1987), no. 6, 30-35.

[30] V. N. Sorokin, On the irrationality of the values of hypergeometric functions, Mat. Sb. (N.S.) 127 (1985), 245-258; English transl.: Math. USSR-Sb. 55 (1986), 243-257.

[31] T. Stihl, Arithmetische Eigenschaften spezieller Heinescher Reihen, Math. Ann. 268 (1984), no. 1, 21-41.

[32] K. Väänänen, On linear forms of a certain class of $G$-functions and p-adic $G$-functions, Acta Arith. 36 (1980), 273-295.

[33] K. Väänänen and G. S. Xu, On linear forms of G-functions, ibid. 50 (1988), 251-263.

[34] O. N. Vasilenko, Linear independence of values of certain hypergeometric functions, Vestnik Moskov. Univ. Ser. I Mat. Mekh. 1985, no. 5, 34-37; English transl.: Moscow Univ. Math. Bull. 40 (1985), no. 5, 40-43.

[35] Q. Wu, On the linear independence measure of logarithms of rational numbers, Math. Comp. 72 (2003), 901-911.

Ville Merilä

Department of Mathematics

University of Oulu

PO Box 3000

90014 Oulun Yliopisto, Finland

E-mail: vmerila@sun3.oulu.fi

Received on 20.8.2009

and in revised form on 18.1.2010 